\title{
Editorial: Role of Mitochondrial Quality Control in Myocardial and Microvascular Physiology and Pathophysiology
}

\author{
Amanda Lochner ${ }^{1}$, Hsueh-Hsiao Wang ${ }^{2}$, Russel J. Reiter ${ }^{3}$, Rui Guo ${ }^{4}$ and Hao Zhou ${ }^{5 *}$ \\ 'Department of Biomedical Sciences, Faculty of Health Sciences, University of Stellenbosch, Stellenbosch, South Africa, \\ ${ }^{2}$ Department of Medicine, Mackay Medical College, Taipei, Taiwan, ${ }^{3}$ The University of Texas Health Science Center at San \\ Antonio, San Antonio, TX, United States, ${ }^{4}$ College of Life Sciences, Hebei University, Baoding, China, ${ }^{5}$ Department of \\ Cardiology, Chinese People's Liberation Army General Hospital, Beijing, China
}

Keywords: mitochondrial fission, mitochondrial fusion, mitochondrial biogenesis, mitophagy, mitochondriadependent cell death, endothelial cells, cardiomyocytes

\section{Editorial on the Research Topic}

Role of Mitochondrial Quality Control in Myocardial and Microvascular Physiology and Pathophysiology

Mitochondrial quality control (MQC) involves a series of adaptive responses of mitochondrial morphological alterations and functional modifications, such as mitochondrial fusion, mitochondrial fission, mitophagy, mitochondrial biogenesis, mitochondrial bioenergetics, and mitochondria-mediated death pathways (Akbari et al., 2019; Del Campo, 2019; Shanmughapriya et al., 2020; Wang et al., 2020c). Mitochondrial damage or impaired MQC has been reported to play an important role in regulating the physiology and/or pathology of myocardium and vessels (Heusch, 2019; Hughes et al., 2020; Wang and Zhou, 2020; Wang et al., 2020b). The objective role of the Research Topic "Role of Mitochondrial Quality Control in Myocardial and Microvascular Physiology and Pathophysiology" (https:/www.frontiersin.org/research-topics/ 13532/role-of-mitochondrial-quality-control-in-myocardial-and-microvascular-physiologyand-pathophysiology\#research-topic-overview) was to gather original research articles and/or reviews to highlight the recent findings regarding the impact of MQC on various cardiovascular disorders. The article "Physical exercise: a novel tool to protect mitochondrial health" by Sorriento et al. reviews the effects of physical activity on cardiac mitochondrial function underlying the ability to modulate specific steps in mitochondrial quality control in both physiological and pathophysiological conditions. Topics were discussed ranged from the effects of exercise on mitochondrial phenotypes, biogenesis, turnover, morphology and respiration to cardiac pathophysiological conditions such as, aging, ischemia/reperfusion injury (I/R), diabetic cardiomyopathy, and anthracyclines dependent heart failure. From these studies, physical exercise emerges as a non-pharmacological tool ("mitochondrial medicine for muscle") to improve cardiovascular fitness in healthy people as well as to attenuate mitochondrial dysfunction in patients with pathophysiological conditions, particularly cardiac I/R damage.

Although several critical molecules of mitochondrial quality control have been identified to improve their function, a drug that specifically targets mitochondria has yet to be developed (Jusic and Devaux, 2020; Larson-Casey et al., 2020; Martínez-Milla et al., 2020; Wang et al., 2020a). A number of promising mitochondria-targeted agents have been studied during myocardial I/R, but none of these exhibited sufficient efficacy for clinical use (Hohendanner and Bode, 2020; Ni et al., 2020; Paik et al., 2020; Zhou et al., 2020). The role of the SERCA2a/Ca ${ }^{2+}$-Mfn2 pathway in myocardial ischemia was investigated by Tian and Zhang. 
By isolation of cardiac microvascular endothelial cells (CMECs) from heart tissues, they found that hypoxia induced irreversible oxidative modifications of SERCA2a, cytosolic and mitochondrial $\mathrm{Ca}^{2+}$ overload, mPTP opening and membrane potential disruption were attenuated by either SERCA2a overexpression or Mfn2 ablation. Mfn2 knockout also suppressed mitochondrial fission and Parkin/PINK dependent mitophagy. Thus, their study showed that ablation of Mfn2 rendered the heart resistant to ischemic injury, reduced cardiac microcirculatory damage, suggesting that Mfn2 inhibition during acute myocardial ischemia injury could be a novel cardioprotective strategy. In contrast to this finding, Liu et al. further observed that Mfn2 overexpression was able to attenuate cardio-cerebrovascular ischemia/reperfusion injury through activation of mitochondrial fusion in a manner dependent on the AMPK/Sirt3 pathway. The beneficial actions of Mfn2controlled MQC were also confirmed by Xiao et al. in a model of hyperglycemia in cardiomyocytes.

In addition to mitochondrial fission or fusion, the role of mitophagy was also discussed by Li et al. in a model of high-fatinduced endothelial dysfunction. They reported that activation of Bnip3-related mitophagy was associated with decreased mitochondrial oxidative stress and increased mitochondrial bioenergy production. Similar to these findings, Xin et al. reported that hypoxia-mediated cardiomyocyte damage could be attenuated by Opa1-related mitophagy through improving MQC. Lastly, in a review summarized by Chang et al.

\section{REFERENCES}

Akbari, M., Kirkwood, T. B. L., and Bohr, V. A. (2019). Mitochondria in the signaling pathways that control longevity and health span. Ageing Res. Rev. 54:100940. doi: 10.1016/j.arr.2019.100940

Del Campo, A. (2019). Mitophagy as a new therapeutic target for sarcopenia. Acta Physiol. 225:e13219. doi: 10.1111/apha.13219

Heusch, G. (2019). Coronary microvascular obstruction: the new frontier in cardioprotection. Basic Res. Cardiol. 114:45. doi: 10.1007/s00395-019-0756-8

Hohendanner, F., and Bode, D. (2020). Mitochondrial Calcium in heart failure with preserved ejection fraction-friend or foe? Acta Physiol. 228:e13415. doi: 10.1111/apha.13415

Hughes, W. E., Beyer, A. M., and Gutterman, D. D. (2020). Vascular autophagy in health and disease. Basic Res. Cardiol. 115:41. doi: 10.1007/s00395-020-0802-6

Jusic, A., and Devaux, Y. (2020). Mitochondrial noncoding RNAregulatory network in cardiovascular disease. Basic Res. Cardiol. 115:23. doi: $10.1007 /$ s00395-020-0783-5

Larson-Casey, J. L., He, C., and Carter, A. B. (2020). Mitochondrial quality control in pulmonary fibrosis. Redox Biol. 33:101426. doi: 10.1016/j.redox.2020.101426

Martínez-Milla, J., Galán-Arriola, C., Carnero, M., Cobiella, J., Pérez-Camargo, D., Bautista-Hernández, V., et al. (2020). Translational large animal model of hibernating myocardium: characterization by serial multimodal imaging. Basic Res. Cardiol. 115:33. doi: 10.1007/s00395-020-0788-0

Ni, L., Yuan, C., Chen, G., Zhang, C., and Wu, X. (2020). SGLT2i: beyond the glucose-lowering effect. Cardiovasc. Diabetol. 19:98. doi: 10.1186/s12933-020-01071-y

Paik, D. T., Chandy, M., and Wu, J. C. (2020). Patient and disease-specific induced pluripotent stem cells for discovery of personalized cardiovascular drugs and therapeutics. Pharmacol. Rev. 72, 320-342. doi: 10.1124/pr.116.013003

Shanmughapriya, S., Langford, D., and Natarajaseenivasan, K. (2020). Inter and Intracellular mitochondrial trafficking in health and disease. Ageing Res. Rev. 62:101128. doi: 10.1016/j.arr.2020.101128

Wang, J., Toan, S., Li, R., and Zhou, H. (2020a). Melatonin fine-tunes intracellular calcium signals and eliminates myocardial damage through the IP3R/MCU discussed the potential natural drugs targeting MQC in the treatment of cardiovascular disorders. Natural medicines or Chinese herbal medicines have special advantages in the treatment of cardiovascular diseases through multiple and complex mechanisms. This review expands our perspectives and promotes the development of new tools or compounds for future preventive and therapeutic strategies in order to reduce the adverse cardiovascular events. Besides, Chang et al. depicts a promising field that places the interaction between MQC and natural drugs at the forefront of the cardioprotection field to extend lifespan.

In summary, these articles and reviews presented in the Research Topic lay a foundation for us to better understand the role of MQC in myocardial and microvascular pathophysiological conditions. This may highlight a new entry point for treating cardiovascular diseases by targeting MQC.

\section{AUTHOR CONTRIBUTIONS}

AL, H-HW, and RR: conceptualization. AL, RG, and HZ: writing and original draft preparation and writing review and editing. All authors contributed to the article and approved the submitted version.

\section{FUNDING}

This work was supported in part by the NSFC (No. 81900252).

pathways in cardiorenal syndrome type 3. Biochem. Pharmacol. 174:113832. doi: $10.1016 /$ j.bcp.2020.113832

Wang, J., Toan, S., and Zhou, H. (2020b). Mitochondrial quality control in cardiac microvascular ischemia-reperfusion injury: New insights into the mechanisms and therapeutic potentials. Pharmacol. Res. 156:104771. doi: 10.1016/j.phrs.2020.104771

Wang, J., Toan, S., and Zhou, H. (2020c). New insights into the role of mitochondria in cardiac microvascular ischemia/reperfusion injury. Angiogenesis 23, 299-314. doi: 10.1007/s10456-020-09720-2

Wang, J., and Zhou, H. (2020). Mitochondrial quality control mechanisms as molecular targets in cardiac ischemia-reperfusion injury. Acta Pharm. Sin. B 10, 1866-1879. doi: 10.1016/j.apsb.2020.03.004

Zhou, H., Toan, S., Zhu, P., Wang, J., Ren, J., and Zhang, Y. (2020). DNAPKcs promotes cardiac ischemia reperfusion injury through mitigating BI-1-governed mitochondrial homeostasis. Basic Res. Cardiol. 115:11. doi: 10.1007/s00395-020-0786-2

Conflict of Interest: The authors declare that the research was conducted in the absence of any commercial or financial relationships that could be construed as a potential conflict of interest.

Publisher's Note: All claims expressed in this article are solely those of the authors and do not necessarily represent those of their affiliated organizations, or those of the publisher, the editors and the reviewers. Any product that may be evaluated in this article, or claim that may be made by its manufacturer, is not guaranteed or endorsed by the publisher.

Copyright (c) 2021 Lochner, Wang, Reiter, Guo and Zhou. This is an open-access article distributed under the terms of the Creative Commons Attribution License (CC $B Y)$. The use, distribution or reproduction in other forums is permitted, provided the original author(s) and the copyright owner(s) are credited and that the original publication in this journal is cited, in accordance with accepted academic practice. No use, distribution or reproduction is permitted which does not comply with these terms. 